\title{
CIRED 2017 - Internationale Konferenz über elektrische Verteilnetze
}

W. Tenschert OVE

Online publiziert am 20. November 2017

(C) Springer-Verlag GmbH Austria, ein Teil von Springer Nature 2017

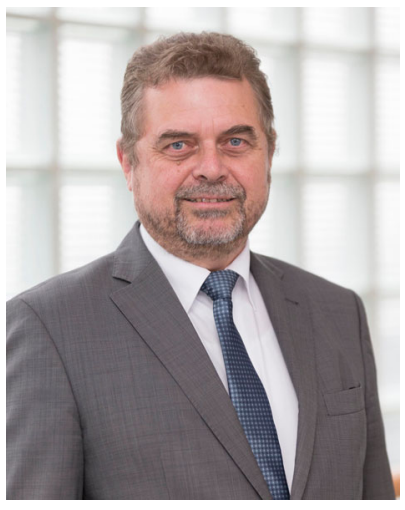

Dipl.-Ing. Dr. Walter Tenschert
Alle zwei Jahre findet die internationale Konferenz der CIRED über elektrische Verteilnetze statt. Die Konferenz bietet eine ausgezeichnete Plattform zu allen technischen Fragen in Verteilnetzen, dem aktuellen Stand der Technik sowie der laufenden Forschungsund Entwicklungstätigkeit. Nach 2015 in Lyon fand die 24. CIRED-Konferenz vom 12. bis 15. Juni $2017 \mathrm{im}$ Scottish Event Campus SEC in Glasgow statt.

1.638 Teilnehmer an der Konferenz sowie an den Ausstellungen aus 54 Ländern nutzten die vier Tage in Glasgow zum Wissens- und Erfahrungsaustausch. Aus Österreich waren 33 Teilnehmer gemeldet. Von mehr als 1.300 zur Konferenz eingereichten Beiträgen wurden 859 Beiträge in einem umfassenden Evaluierungsprozess, in den auch zahlreiche österreichische Experten eingebunden waren, akzeptiert. Die Anzahl eingereichter österreichischer Beiträge ging zwar etwas zurück, die Anzahl akzeptierter Beiträge blieb aber mit 20 verglichen mit den letzten Konferenzen auf gleichem Niveau.

Die traditionelle Gliederung der Konferenz in sechs fachliche Teilbereiche (Sessions) wurde auch 2017 beibehalten:

1) Netzkomponenten

2) Spannungsqualität und elektromagnetische Verträglichkeit

3) Betrieb, Steuerung und Schutz

4) Dezentrale Energieressourcen und aktive Verbrauchsintegration

5) Verteilnetzplanung

6) Geschäftliches Umfeld

Am ersten Tag fanden noch vor der offiziellen Eröffnung sechs Tutorials zu ausgewählten Themen in Verteilnetzen statt. An den drei Haupttagen der Konferenz wurden die Beiträge der einzelnen Sessions präsentiert. Dafür standen teilweise zeitgleich abgehaltene Plenum-Vorträge, Round-table-Diskussionen, Forschungs- und Innovationsforen sowie Poster-Sessions statt. Insbesondere die PosterSessions, bei denen die Autoren ihre Beiträge im direkten Gespräch mit interessierten Experten erläutern konnten, fanden großen Anklang. Die Fachbeiträge wurden von einer umfassenden Ausstellung über verteilnetzrelevante Produkte namhafter Hersteller ergänzt. Im Anschluss an die Konferenz konnten wieder technische Anlagen in der Umgebung von Glasgow besichtigt werden. Dieses Jahr wurden die Besichtigung eines Windkraftwerksparks sowie des Power
Networks Demonstration Centre der University of Strathclyde angeboten.

Die auch dieses Jahr überproportionale Beteiligung Österreichs an der Konferenz ist auf die aktive Rolle der österreichischen Universitäten, Forschungsinstitute, Industrie und Netz- bzw. Kraftwerksbetreiber zurückzuführen. An dieser Stelle ein herzliches Dankeschön an alle, die dazu beitragen, Österreichs Bedeutung auf dem Gebiet der elektrischen Verteilnetze zu stützen.

Im Anschluss an die Konferenz wurde in der Sitzung des Directing Committees die periodisch geplante Veränderung der Leitung der CIRED beschlossen. Theodor Connor (Deutschland) übergab den Vorsitz des Directing Committees an den bisherigen Leiter des Technical Committees Emmanuel de Jaeger (Belgien), der wiederum seine bisherige Aufgabe an Pierre Mallet (Frankreich) übergab.

Für diejenigen, die an der Konferenz nicht teilnehmen konnten, bietet das österreichische Nationalkomitee wieder zwei InfoNachmittage mit Präsentationen über die aktuellen Trends der sechs Sessions an:

\section{Wien, Dienstag, 30. Jänner 2018}

in den Räumlichkeiten des OVE, Eschenbachgasse 9,

1010 Wien

Innsbruck, Dienstag, 20. Februar 2018

Hotel Grauer Bär, Universitätsstraße 5-7, 6020 Innsbruck

Nächste CIRED-Veranstaltungen:

CIRED Workshop 2018 - Ljubljana, Slowenien

7.-8. Juni 2018, Cankarjev Dom

Microgrids and local energy communitites

CIRED Conference 2019 - Madrid, Spanien

3.-6. Juni 2019, Feria de Madrid (IFEMA)

Die nachfolgenden Beiträge über die CIRED-Sessions stellen eine Kurzfassung der Inhalte und Tendenzen dar und sollen zu einer intensiveren Befassung mit den Beiträgen der CIRED-Konferenz anregen.

Weiterführende Informationen oder Ansprechpartner finden Sie auf den Websites:

http://www.cired.at

http://www.cired-2017.org

http://www.cired2018-workshop.org

http://www.cired2019.org
Tenschert, Walter, Salzburg Netz GmbH, Bayerhamerstraße 16, 5020 Salzburg, Österreich (E-Mail: Walter.Tenschert@salzburgnetz.at) 